\title{
A Data-driven Agent Based Simulation platform for Early Health Economics Device Evaluation
}

\author{
David Bell \\ Dept. of Computer \\ Science \\ Brunel University \\ London
}

\author{
Armin Kashefi \\ Dept. of Computer \\ Science \\ Brunel University \\ London
}

\author{
Nurul Saleh \\ Dept. of Computer \\ Science \\ Brunel University \\ London
}

\author{
Tommaso Turchi \\ Dept. of Computer \\ Science \\ Brunel University \\ London
}

\begin{abstract}
Health economics is a relatively new but growing field within the discipline of economics and is concerned with making the best use of scarce resources. Early health economic estimates of new medical devices, in particular, can assist producers of health technology in making appropriate product design and investment decisions. It allows companies to understand their likely market and possible reimbursement more thoroughly. Despite the many advantages of point-of-care testing the key problem facing decision makers at the moment is the poor understanding of the potential value gained from new or alternative product or service offerings. Understanding medical device features in the wider market place can be addressed used agent based modelling and simulation (ABMS). In this paper we examine the use of ABMS underpinned by a novel datadriven approach to model generation. A sepsis use case is presented where pathway and device characteristics are defined using the 'headroom' method and a semantic evidence capture application. Types and sub-types are automatically extracted into agent models and subsequently executed in our own data-driven agent based simulation platform (TEASIM). A highly typed data-driven approach is evaluated in a manner that clearly presents the technical aspects of TEASIM platform and its practical usage. Initial evaluation of a data-driven approach (and the TEASIM platform) is positive. The approach offers a viable guide to product development in a cost-effective manner, especially in the earlier stages when deciding between potential product configurations or features.
\end{abstract}

\section{Author Keywords}

Health economic assessment, agent based modelling and simulation (ABMS), headroom method, cost effectiveness analysis

\section{ACM Classification Keywords I.6.1 SIMULATION AND MODELING (Model Development).}

\section{INTRODUCTION}

Health technologies continue to make a substantial contribution to improving the health status of populations [1]. Nonetheless, OECD countries over a period of 10 years have witnessed an annual average health expenditure growth per capita of $4 \%$, outpacing economic growth of $2.2 \%$ during the same period [2]. A consequence of this is that health systems are faced with an economic problem and continually looking to achieve more for less economic cost. The medical device industry and device developers have an important part to play in ensuring that efficiencies are achieved through increased efficiency of spending, and not at the expense of the quality of care received by patients [3]. One solution would be to enhance the decision maker understanding of the medical device decision domain and subsequent quality of go/no-go product development choices by incorporating health economic assessments in such decision-making processes $[3,4,5]$. The economic evaluation of public health interventions, in essence, provides a capability to maximize population health subject to scarce resources $[6,7]$. This makes it especially important and relevant to diagnostic services and Point-of-Care (PoC) technologies [8]. This is typically carried out

The PoC diagnostic sector, which is a growing market, creates a challenging evaluative scenario because, on the one hand, the unit cost-per-test is larger through the loss of the economies of scale offered by automation, whilst it offers the potential of substantial savings through enabling rapid delivery of results, and reduction of facility costs [9]. In this context, allowing PoC builders to quickly design increasingly 'realistic' representations of what the product should do with a reasonable reimbursement price is not only a critical area of research but also a challenging one due to the fact that the properties of PoC devices closely interrelate with varied technical and non-technical healthcare information needs. Knowledge platforms are able to support the collection and synthesis of healthcare information supporting economic evaluation - typically using aggregate measures and population data. Coupling knowledge platforms with agent modelling provide decision makers with a novel combination of device re-imbursement and system impact analysis. 
Adopting a design-science paradigm, with an emphasis on problem-solving, we introduce a number of innovative artefacts in order to design novel data-driven decisionmaking tools - aimed at improving the early health economic evaluations of PoC devices and their subsequent usage. Artefacts are built as part of a design-build-evaluate cycle towards an effective solution and as theoretical knowledge is accumulated. The paper presents two designbuild-evaluation cycles that result in knowledge (TeaPoCT) and agent modelling (Tea-Sim) platforms for early health economic evaluations by $\mathrm{PoC}$ device manufacturers. Importantly, the transition from knowledge platform to agent model is a primary focus in this paper. The structure of the paper is as follows: Section 2 presents work related to our efforts in economic evaluation and agent based modelling. In Section 3 our knowledge capture and annotation approach is detailed. Section 4 presents agent modeling driven by the knowledge platform. Section 5 presents an evaluation of the resulting design artefacts, conclusions and opportunities for future work.

\section{BACKGROUND}

Health Economics is a relatively new but growing field within the discipline of economics and is concerned with making the best use of scarce resources [10-12]. It has been an established feature of research, policymaking, practice and management in the delivery of healthcare [[9] and its role is increasing as the cost of healthcare begins to drive changes in most major healthcare systems. Economic modelling, simulation and scenario analysis are tools that help decision makers make choices in a rational fashion [13]. This emerging area has consequently become the focus of both public policy and private sector transactions with associated input and output, input being healthcare spending and output being improved outcomes for patients $[14,15]$. A relevant case for health economics is the National Health Service (NHS) in the UK as it has been acknowledged that increases in NHS spending were not being accompanied by markedly improved outcomes [16]. Consider the NHS, 'Health Economic' evaluation offers decision-makers the tools to maximize output relative to a fixed budget input.

Value assessment for medical products is a complex task and developing products or investing in this sector can be a daunting process $[17,18]$. If the ultimate goal of healtheconomic evaluation of medical interventions is to support evidence-based policy decisions, then it is essential to place new products in terms of cost and effectiveness, relative to the current gold standard treatment [3].

The 'Headroom method' is used to assess the costeffectiveness of a new technology in comparison to some existing gold standard technology, by defining a maximum cost (the Headroom) that the adopter of the technology would be willing to incur [19]. Technologies or projects whose cost exceeds the maximum Headroom can be deemed cost-ineffective and abandoned. On the other hand, technologies whose costs fall below the Headroom are not necessarily going to be cost-effective, but they show potential for adoption, which can be decided at later stages of the cost-effectiveness assessment. Therefore, the method can be used as a barrier to unsustainable projects at early stages, or even before their commencement [20]. The Headroom method offers a simple way for manufacturers to investigate the commercial viability of a new medical device, by applying the principles of health economic evaluation early on, ideally at the concept stage [20]. Clinical data verifying effectiveness is apparently absent at this stage, but the innovator's ideas around what the device could mean for patients (impact on health) and the NHS (cost impact for services) can be used to estimate its reimbursement prospects. Thus, the method uses early predictions of potential impact (which should be optimistic but plausible) to estimate what the NHS should be willing to pay for the device if it works as hoped. In short, the Headroom method can be explained as follows: [20]

Agent based modelling (ABM) has been applied in a number of domains including economics [21], health-care [22], and geography [23] among others. ABM also has links with other disciplines including social sciences, artificial life science, management science and complexity science $[24,25]$. A resurgence in interest in ABM likely benefits from the most recent development in the Artificial Intelligence (AI), and Individual Based Modelling (IBM) in ecology. More traditionally, ABM has been applied to cellular automata to investigate housing segregation patterns by modelling people and the socially relevant process, which represent interactions between people [26]. In business, agent-based modelling has been applied to help decision makers understand underlying market structures and anticipate dynamics in the market place [25]. Simulations can help clarify uncertainty by identifying the causes of the market dynamics.

$\mathrm{ABM}$ is often a process comprising analysis, design, implementation, execution and validation [27]. State charts often underpin the ABM approach and more specifically an understanding of environmental and agent state [28] typically investigating the expenses associated with a healthcare setting. State machine inputs, outputs and probabilistic state transition drive the executing simulation [29]. ABM in healthcare has traditionally been motivated by the analysis and construction of models [28] that rely heavily on domain experts for their construction. In contrast, relying purely on the data generated by a realworld health systems is more ambitious. However, the growth in open data is providing a means to uncover insights from this type of novel approach. Emergency departments have been a popular area to investigate, better understanding the modelling required [27] and subsequent optimization [29]. Synthesizing the economic modelling with agent approaches (especially for medical device manufacturers) has had less coverage in the literature. 


\section{LANGUAGE, STYLE AND CONTENT}

The design problem being addressed is accessibility to data and decision making tools for the medical device manufacturers. Our data-driven simulation design response combines a knowledge capture platform with agent based simulation. The remainder of this section describes the first three steps before focusing on the agent modelling in the following section.

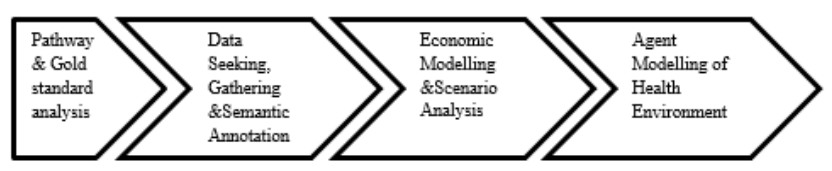

Figure 1. Data-driven Simulation Framework

The remainder of this section describes the first three steps before focusing on the agent modelling in the following section.

\subsection{Knowledge Capture Platform}

We use a Sepsis scenario to demonstrate our approach. Initially, a first data model is created, recording Sepsis facts on our Tea-PoCT platform. MediaWiki software was extended to support visual and semantic tagging and hosted at www.Tea-PoCT.com. MediaWiki (or Wiki technology) was chosen at it offers functionality to store several revisions of the same file, made by different users, and provides a place to store and validate emerging evidence. Secondly, it provides a basis by which an analyst can turn validated information residing in the wiki into structured information for later use. MediaWiki is also a full-fledged development framework (PHP), with available extensions (i.e. semantic MediaWiki, html tags) that can turn a wiki into a powerful and flexible knowledge management system. All data created within Tea-PoCT can then be accessible on the Semantic Web, allowing other applications to use this data seamlessly (due in part to the self-describing nature of the platform). Figure 2 shows two snapshots of the Tea-PoCT wiki, one from the main page and one from the Sepsis page.

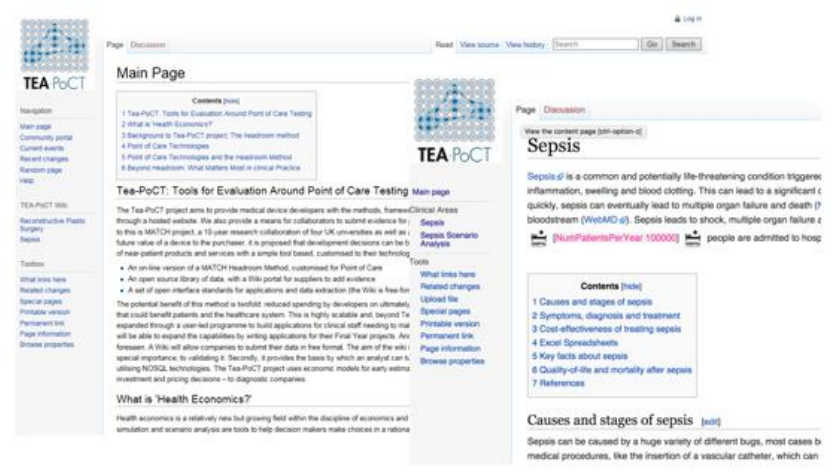

Figure 2. Snapshots of the Tea-PoCT MediaWiki
To provide accessible and consumable data for platform services and $\mathrm{PoC}$ device manufacturers, an extractor was developed for accessing wiki stored clinical data. The aim of the extractor is to harvest the 'semantically annotated data' ready for the agent platform or other external services. Semantically annotated data is able to be read and understood not only by human but also by machines. The main point in creating semantically annotated data is enabling applications to search, retrieve and process the content in a more intelligent way. Authorised clients can then download this data into the Agent platform, Excel spreadsheet or Web based economic modelling for device specific analysis.

Once the Tea-PoCT platform became available, an extensive literature review was undertaken to collect data about the clinical area under investigation.

\subsection{Knowledge Gathering}

Currently the key problem facing $\mathrm{PoC}$ decision makers is lack of access to good evidence and clear cases concerning product or service data. A comprehensive literature review is therefore needed to identify product data sources and to summarize the existing evidences on four clinical areas being explored (Sepsis). Sepsis is one of the most expensive conditions that can affect patients. In the UK alone, the Sepsis Trust puts NHS spending linked to the condition at $£ 2.5$ billion (NCEPOD, 2014). The diagnosis of this condition is quite complicated as it appears like a range of other medical conditions in its early stages. For clinicians that have seen Sepsis develop before, they may be able to diagnose it with successful treatment during the early stages. Nevertheless, for clinicians without prior direct experience with Sepsis, the diagnosis is more problematic. Although Health Economic (HE) basics are relatively easy to grasp, accessing quality data quickly, is however problematic. Data will often not exist - especially if the product is novel and so appropriate data must be inferred from the literature, elicited from experts or even estimated. Once identified, product and service data is semantically annotated and compiled into Tea-PoCT using 'PicTags' (or picture tags). PicTags are small icons that have a unique name, associated data (e.g. cost, price and charge), links and sematic annotation. With the help of PicTags the key names and values can be differentiated from the surrounding text and can be identified and extracted through semantic mechanisms and queries. Figure 3 illustrates the semantically annotated data in our MediaWiki system. In addition, MediaWiki was extended to provide a secure means of safeguarding the data from unauthorized access. Accordingly, unauthenticated users (i.e. public view) cannot read or understand the semantically annotated data that are crucial for costeffectiveness analysis. 


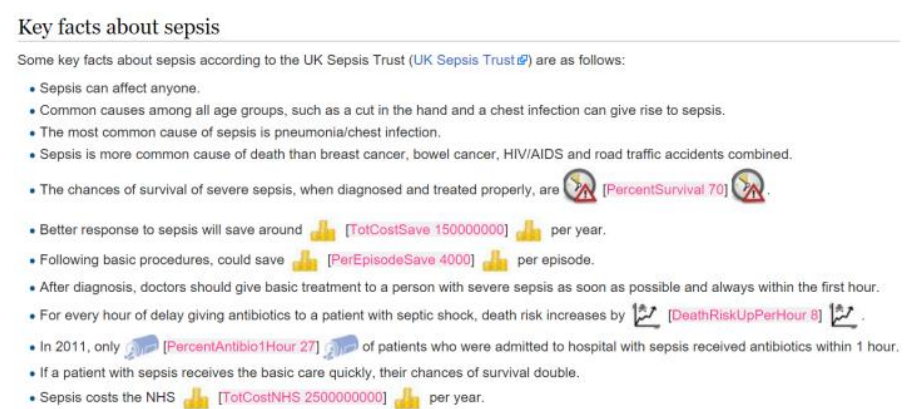

Figure 3. Semantically annotated data with pictags

Once data is captured and semantically annotated, economic and scenario modelling can be carried out.

\subsection{Knowledge Gathering}

At this stage an economic model was constructed within Tea-PoCT, based on the Headroom method, in order to explore various Sepsis cost-effectiveness scenarios. The economic model was developed in such a way as to evaluate and predict the cost-effectiveness of PoC devices with specific functionalities, quality-adjusted life years (QALYs) and the Headroom over time periods up to 6 years, which can be extended as required. The rationale is to allow decision maker to interact with the data directly and to explore different development consequences. We believed that this approach would further help $\mathrm{PoC}$ developers to attain a better understanding of the early health economic evaluations and the challenges they might face. Unsurprisingly, this same data can be easily utilized uncovering agents and their behaviors. Furthermore, interested parties are able join the Tea-PoCT project and provide further input into the modelling and to make the prediction and economic assessments even more finegrained. In our economic model the Headroom is formulated as follows:

$\mathrm{MRP}=\mathrm{P} 2=\mathrm{WTP}$ threshold $\mathrm{x} \Delta \mathrm{QALY}-\Delta \mathrm{SC}+\mathrm{P} 1$

Where:

- $\quad \mathrm{MRP}=$ Maximum reimbursable price

- $\quad \mathrm{P} 2=$ The maximum price the manufacturer can charge for the new device (=MRP)

- WTP threshold = Pre-determined QALY tariff between $£ 20,000$ and $£ 30,000$ per patient

- $\triangle \mathrm{QALY}=$ Increments in effectiveness between the new device and the gold standard, measured in QALYs. $\triangle \mathrm{QALY}=$ (utility score of treatment B $\mathrm{x}$ duration (years) of that health state) - (utility score of treatment $\mathrm{A} x$ duration (years) of that health state)

- $\Delta \mathrm{SC}=$ Decrements or savings in service costs due to the new more effective device. $\triangle \mathrm{SC}=$ Cost of
Treatment B (New tech) - Cost of Treatment A (Old/Gold standard)

- $\quad \mathrm{P} 1=$ The price of the old device (if it exists)
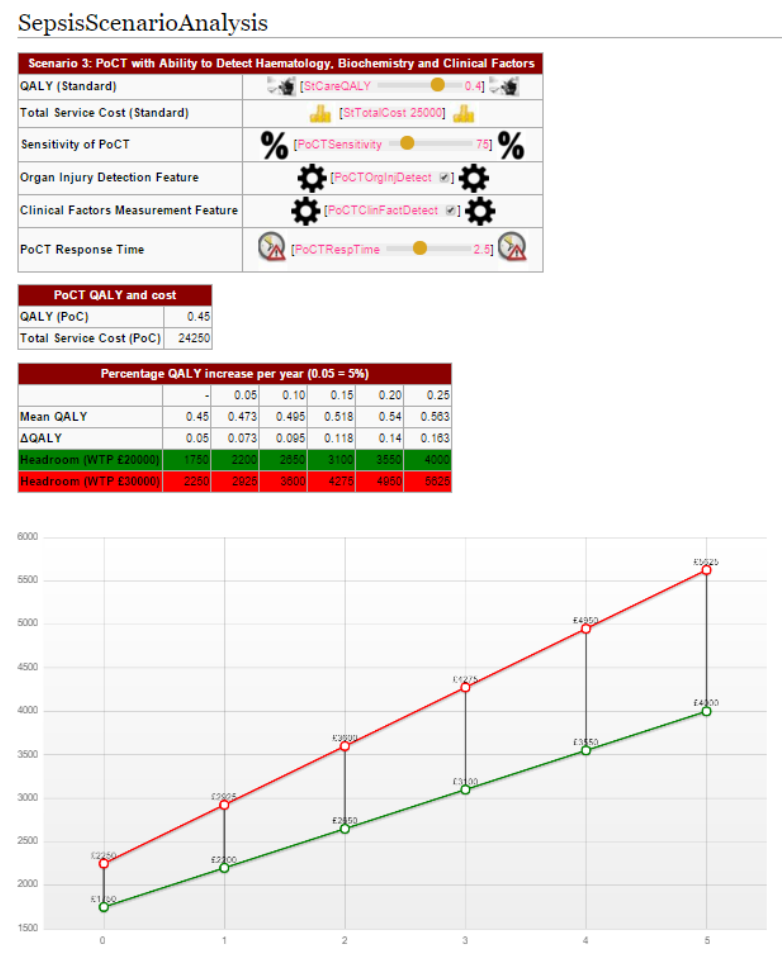

Figure 4. Scenario Analysis

Figure 4 represents different characteristics of a $\mathrm{PoC}$ device, allowing the user to change the highlighted fields and experience different cost-effectiveness and development scenarios. The headroom method is implemented within the wiki page alongside scenario specifications. Tables and graphical results are also defined (again as pictags) and presented within the page. Scenarios can be tested to fully understand the economic implications of a medical device and its usage. Now that the device manufacturer understands the economic impact of the device, the same data can then be used for simulating it use in the healthcare environment.

\section{AGENT MODELLING}

The next design challenge was how best to reuse the captured and validated data within a simulation environment - extracting appropriate pictag data of value. Our first design instantiation (presented in this paper) focuses on the agent modelling paradigm. A number of agent modelling environments exist, but tend to be either graphical based, where the user builds a model inside the tool, or programming language based. In contrast, with a rich data repository it was decided to explore how the agent model could more easily be driven by the data itself. Underpinning our simulation approach is a relational model with tables as agent types and rows represented agent instances. This approach enables nuanced variation of 
agent properties (e.g. age) without the need to define a new agent. The executing simulator user JSON files (extracted from a relational model, Tea-PoCT or hand-crafted) to specify agents and behaviors. This form of modelling enables decision makers to gain additional insight into a complex system behavior and to demonstrate the real situation of a sepsis patient in a hospital. A simulation is constructed from available Tea-PoCT pathway or economic data. The simulation is designed to determine the estimated cost of sepsis patient based on their condition and the duration of stay at the hospital. Our data-driven simulation platform is called Tea-Sim and implemented in PHP with supporting JSON files. Both file types can be generated from Tea-PoCT (or other data sources such as relational databases). PHP configuration, agents and agent behavior can make use of Tea-PoCT data to further explore medical device within a health context, e.g. cost impact to a hospital. We have developed a proof of concept model in this paper, but envisage that the same approach can be extended to apply the same medical device economics in a more specific hospital, area or country.

Our hospital environment requires a number of agents to be implemented:

A critical_patient of class people,

Anot_critical_patient of class people,

A dead_patient of class people,

A normal_patient of class people and

A nurse of class people.

In our Tea-Sim platform, each agent is denoted by specific id and set of variables. The agent variables are listed below:

\$this->_supertype

\$this->_type

\$this->_id

\$this->_img

$\$$ step

These variables are accessible to the step functions, including the pointer to a visual representation of the current agents state (_img). Agent behavior is then implemented in a number of available functions, such as:

getPosition()

move(\$distance)

anyNeighbour(\$distance[,\$type[,\$select[,\$update]]])

allNeighbour(\$distance[,\$type[,\$select[,\$update]]])

morph(\$type)

In order to set up the sepsis simulation, two files are required for initialization and model execution - init.json and model.json. JSON file are used as these are easily processed and generated from the Tea-PoCT platform. The init.json file determines the initialization processing to be carried out. Grid size, number of instances for each agent, the position of each agent and the simulation steps are defined in init.json. Agent attributes of class people are defined in model.json with behaviour (step functions) in steps.php.

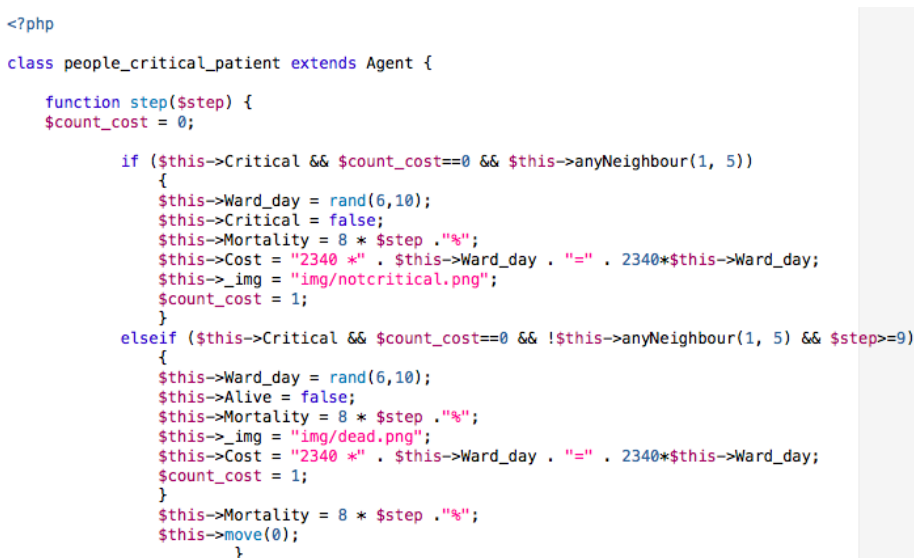

Figure 5. critical_patient of class people agent

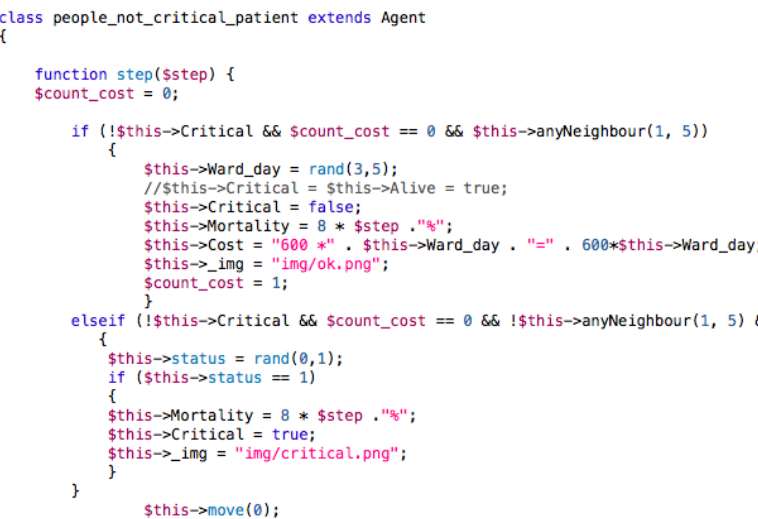

Figure 6. not_critical_patient of class people

Agent behavior specification is described in a module called steps.php. The stepping function, step() describes how each agent of a specific type progresses from one iteration to another, including state changes. In steps.php, the health processes or internal rules are being processed. Figure 5 show the rules applied to critical_patient of class people agent and figure 6 shows the rules applied to not_critical_patient of class people agent.

The processes provided in steps.php describe agent actions, such as:

i. If nurse is not near the not_critical_patient, then make yes/no decision to become critical_patient or not_critical_patient 
i.i. If (not_critical_patient is still not_critical_patient), check if nurse is around

i.i.i. If YES (nurse is around), not_critical_patient become normal_patient,

Probabilistically determine the length of stay in general ward and calculate cost

i.i.ii. If NO (nurse is not around), not_critical_patient become critical_patient

i.ii. If (not_critical_patient is now critical_patient), check if nurse is around

i.ii.i. If YES (nurse is around), probabilistically determine the length of stay in intensive care unit (ICU) and associated calculate cost

i.ii.ii. If NO (nurse is not around and steps is more or equal than 9), critical_patient become dead_patient and store the total number of days and cost

ii. If nurse is not near or near the critical_patient,

ii.i. If YES (nurse is around), critical_patient become not_critical_patient, probabilistically determine the length of stay in ICU ward and calculate cost

ii.ii. If NO (nurse is not around and steps is more or equal than 9), critical_patient become dead_patient and store the total number of days and cost

Executing simulations can be seen in figures 7-9.

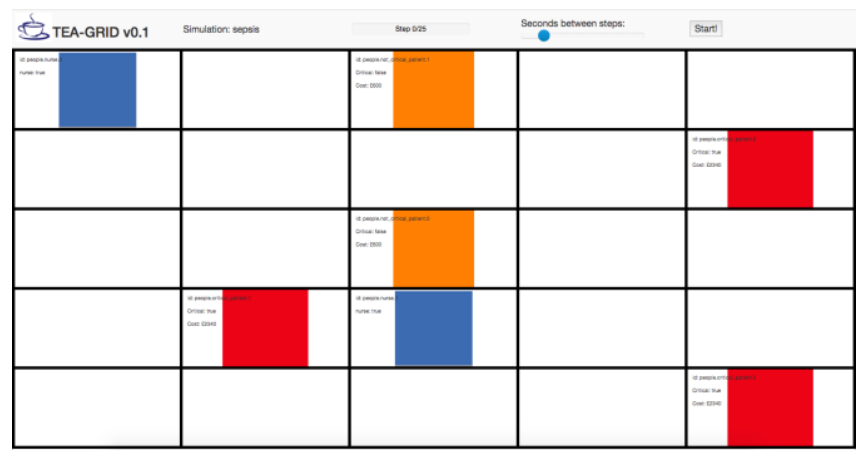

Figure 7. Sepsis Simulation with three types of agent, critical_patient, not_critical_patient and nurse

Figure 7 shows when agent of nurse (blue box) is near agent of critical_patient and agent of not_critical patient it will change the critical_patient to not_critical_patient (green box) and the not_critical_patient to normal_patient and the cost will be calculated (Figure 8). Although we are using simple colored blocks, any image can be used in the simulation with additional state annotation as an option.

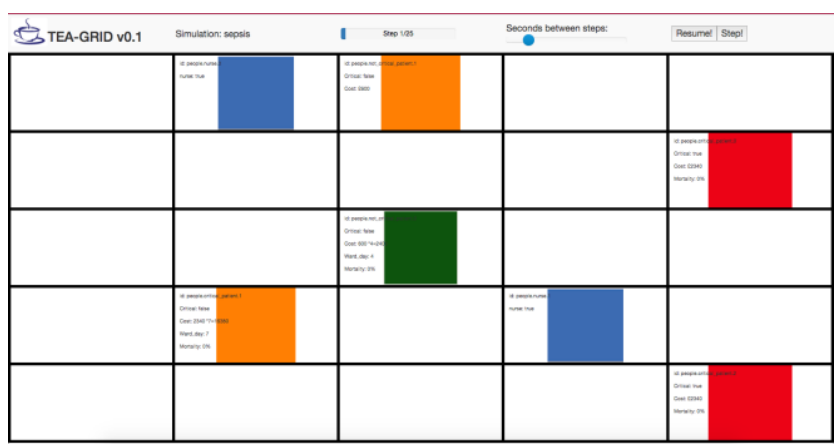

Figure 8. critical_patient become not_critical_patient and not_critical_patient become normal_patient with calculated cost and number of days in ward

In a further simulation, Figure 9, the agent nurse is not near agent critical_patient after the mortality rate reaches $80 \%$ or more, critical_patient become dead_patient (black box)

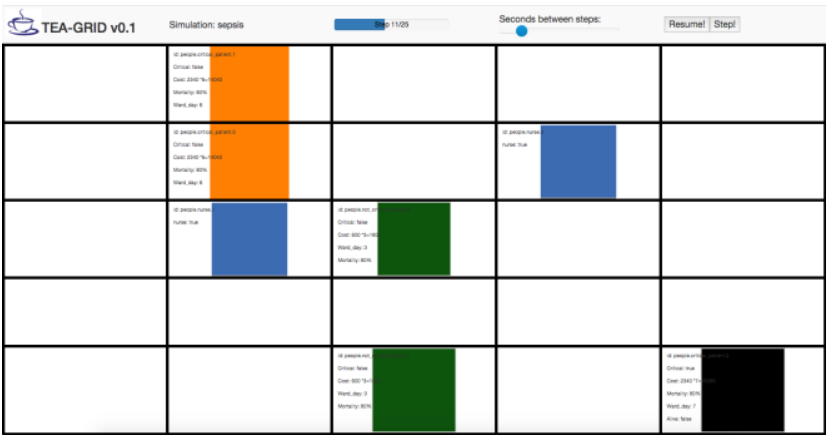

Figure 9. critical_patient become dead_patient after mortality reaches $80 \%$ or more

\section{EVALUATION AND CONCLUSION}

Tea-PoCT and Tea-Sim provide users with the methods, framework and evidence, or simply the basis for making early estimates, through a set of innovative hosted digital services. Tea-PoCT is the first phase of a vision where product and service providers are better connected to patients and near patient practitioners. While the purpose of evaluation in the project's initial phase was to assess the feasibility and effectiveness of the idea of the Tea-PoCT's extended MediaWiki system and the process of data acquisition and economic modelling, the aim of the subsequent design iteration evaluates the utility of economic data to the simulation process. The instantiation of a number of simulations demonstrates the viability of the approach. Furthermore, it was clear that data can be easily re-used in a wider simulation, specifically: 1) Variations of disease, 2) Disease Burden, i.e. mortality rate of $8 \%$ /hour and 3) Economic burden and cost in terms of duration and finance.

This research also opens a number of future research avenues, stimulating improvement in existent processes and further lead to designed advances. It is envisaged that the platform proposed in this paper can further evolve and 
support research into novel client decision-making apps, mobile apps and collaborative economic modelling. Originality in Tea-PoCT and Tea-Sim can also be seen in the bringing together of elements of knowledge seeking and acquisition, modelling and simulation. In addition, a further contribution for the platform in use will be a state of the art medical data vault populated with real world product and service offerings and importantly the associated simulations and scenarios.

Design science is underpinned by the artefacts produced over the course of a design journey, categorized by March and Smith [30] as constructs, models, methods and instantiations. This paper presented a rich set of artefacts, each combining as part of an effective design. Constructs such as pictags provide a means for much of the knowledge identification and re-use. Data models for each disease utilize the technological methods - pictags, extractors, agent code - to drive the agent based simulation models. Tea-PoCT and Tea-Sim instantiations demonstrate the effectiveness of these artefacts and the overall designed approach.

The paper provides a practical insight into how data can drive both the economic modelling of medical devices and their impact on a heath environment. Two design research instantiations are presented - a knowledge acquisition tool Tea-PoCT and agent based simulator Tea-Sim. A sepsis scenario demonstrates how economic modelling data acquisition can be re-used in an agent based simulation.

\section{ACKNOWLEDGMENTS}

We thank Innovate UK for providing the funding for the Tea-PoCT project.

\section{REFERENCES}

1. Grootendorst, P., Piérard, E., \& Shim, M. Lifeexpectancy gains from pharmaceutical drugs: a critical appraisal of the literature, 2009.

2. Simoens, S. Health economic assessment: a methodological primer. International journal of environmental research and public health, 6(12), 29502966, 2009.

3. Chapman, A. M. The use of early economic evaluation to inform medical device decisions: an evaluation of the Headroom method (Doctoral dissertation, University of Birmingham), 2013.

4. Vallejo-Torres, L., Steuten, L., Parkinson, B., Girling, A.J., and Buxton, M.J. Integrating Health Economics into the Product Development Cycle: A Case Study of Absorbable Pins for Treating Hallux Valgus. Medical Decision Making, 31(4): 596-610. 2011.

5. Miller, P. Role of pharmacoeconomic analysis in $R \& D$ decision making: when, where, how?, Pharmacoeconomics, vol. 23, no. 1, pp. 1-12, 2005.
6. Williams, I., McIver, S., Moore, D., \& Bryan, S. The use of economic evaluations in NHS decision-making: a review and empirical investigation, Health Technology Assessment (Winchester, England), vol. 12, no. 7, p. iii. 2008.

7. Carlsson, P. Health technology assessment and priority setting for health policy in Sweden. International journal of technology assessment in health care, 20(01), 44-54, 2004.

8. Edwards, R. T., Charles, J. M., \& Lloyd-Williams, H. Public health economics: a systematic review of guidance for the economic evaluation of public health interventions and discussion of key methodological issues. BMC Public Health, 13, 1001,2013.

9. Sanavio, B., \& Krol, S. On the Slow Diffusion of Pointof-Care Systems in Therapeutic Drug Monitoring. Frontiers in Bioengineering and Biotechnology, 3, 20, 2015.

10. St John, A., \& Price, C. P. Economic Evidence and Point-of-Care Testing. The Clinical Biochemist Reviews, 34(2), 61-74, 2013.

11. Mishra, D., \& Nair, S. R. Systematic literature review to evaluate and characterize the health economics and outcomes research studies, India.Perspectives in Clinical Research, 6(1), 20, 2015.

12. Angevine, P. D., \& Berven, S. Health Economic Studies: An Introduction to Cost-benefit, Costeffectiveness, and Cost-utility Analyses. Spine, 39(22S), S9-S15, 2014.

13. Akeem, B. O., \& Juni, M. H.. Health economics evaluation in health planning. International Journal of Public Health and Clinical Sciences, 2(2), 93-103, 2015.

14. Farag, I., Howard, K., Ferreira, M. L., \& Sherrington, C. Economic modelling of a public health programme for fall prevention. Age and ageing, afu195, 2014.

15. Graves, N., \& Zheng, H. Modelling the direct health care costs of chronic wounds in Australia, 2014.

16. Webber, L., Mytton, O. T., Briggs, A. D., Woodcock, J., Scarborough, P., McPherson, K., \& Capewell, S. The Brighton declaration: the value of non-communicable disease modelling in population health sciences. European journal of epidemiology, 1-4, 2014.

17. Craven, M. The MATCH Project: collaboration between academia and industry, Focus magazine, Association of British Healthcare Industries, July 2004.

18. Girling, A.J., Lilford, R.J., and Young, T.P., Pricing of Medical Devices under Coverage Uncertainty - a Modelling Approach. Health Economics, 21(12), 2012.

19. Davey, S., Brennan, M., Meenan, B., McAdam, R., Girling, A., Chapman, A., and Lilford, R., A, Framework to Manage the Early Value Proposition of 
Emerging Healthcare Technologies. Irish Journal of Management, 31(1), pp.59-75, 2011.

20. Van Nimwegen, K. J. Feasibility of the Headroom Analysis in Early Economic Evaluation of Innovative Diagnostic Technologies with no Immediate Treatment Implications. Value in Health, 17(7), A550, 2014.

21. Farmer J.D. and Foley D. The economy needs agent based modelling. Nature, 460(7256):685-686, 2009.

22.Epstein, J.M.. Modelling to contain pandemics. Nature, 460(7256):687\{687, 2009.

23. Heppenstall, A.J, Crooks A. T., See L.M., and Batty M. Agent-based models of geographical systems. Springer Science \& Business Media, 2011.

24. Ma T. and Nakamori Y. Agent-based modeling on technological innovation as an evolutionary process. European Journal of Operational Research, 166(3):741\{755, 2005.

25. Macal C.M. and North M.J.. Tutorial on agent-based modelling and simulation. Journal of simulation, 4(3): $151\{162,2010$.
26.Li X., Mao W., Zeng D., and Wang F.Y.. Agent-based social simulation and modeling in social computing. Intelligence and Security Informatics, pages $401\{412$. Springer, 2008.

27. Stainsby H, Taboada M, Luque E. Towards an agentbased simulation of hospital emergency departments. Services Computing, IEEE, 2009.

28. Borshchev A, Filippov A. From system dynamics and discrete event to practical agent based modeling: reasons, techniques, tools. Proceedings of the $22 \mathrm{nd}$ international conference of the system dynamics society 2004 Jul 25 (Vol. 22).

29. Cabrera E, Taboada M, Iglesias ML, Epelde F, Luque E. Optimization of healthcare emergency departments by agent-based simulation. Procedia computer science. Dec 31;4:1880-9, 2011.

30. March S.T., Smith G.F.. Design and natural science research on information technology. Decision support systems. 1995 Dec 31;15(4):251-66. 